\title{
Experimental added modal damping induced by confined granular media on a single degree of freedom system
}

\author{
Antoine Sternberger ${ }^{1,2, \star}$, Adrien Pelat ${ }^{2, \star \star}$, and Jean-Michel Génevaux ${ }^{2, \star \star \star}$ \\ ${ }^{1}$ IRT Jules Verne, Chemin du Chaffault, 44340 Bouguenais, FRANCE \\ ${ }^{2}$ LAUM UMR 6613, Université du Maine, Avenue Olivier Messiaen, 72085 Le Mans Cedex 9, FRANCE
}

\begin{abstract}
The use of granular media to induce vibration energy's dissipation in lighter huge industrial structures permits to decrease the mass of the structure and consequently to spare the construction's cost and to satisfy oil consumption. In fact, when the structure in which the granular media is in contact overtakes an acceleration threshold, relative movements of the grains appears which lead to a dissipation of energy. When the grains are confined inside a cavity, the dissipation's level depends on several parameters (the acceleration's amplitude, the frequency, the grain's characteristics, the cavity's dimensions, the cavity's filling ratio, the fluid between the particles, etc.). This study quantifies the influence of several parameters by exciting uniformly a given volume of grains. A modal damping coefficient of a single degree of freedom system (SDOF) can be thus calculated as a function of the preceding parameters.
\end{abstract}

\section{Motivations}

Weight reduction of structure is an important issue for the transport industry in a way of doing some savings. Consequently to this mass reduction, an increase of the vibration is observed. This can lead to a shorter lifetime of the industrial structure by the weakening done trough this evolution.

An original way of structures' damping is the use of granular material. The dissipation is due to the contact between the particles and beween the particles and the surface of the structure. The transmission (and the loss) of the energy has been studied in several cases [1,2]. Numerous advantages of this treatment (little influence of the temperature, easy installation...) make it very attractive for the industry. For example the granular media can be placed inside a cavity which is fixed on the primary system. [3].

Firstly, the mass of the added beads lead to a vibration reduction. This mass effect is completed by damping if relative movement of grains appears inside the granular media. However, this loose media adds a strong non-linear effect which has to be taken into account [4]. The effect of the granular treatment will therefore be influenced by the acceleration's amplitude.

To deduce the damping from the micro-scale of the beads to the macroscopic scale of the structure need

\footnotetext{
^e-mail: antoine.sternberger@irt-jules-verne.fr

$\star \star$ e-mail: adrien.pelat@univ-lemans.fr

$\star \star \star$ e-mail: jean-michel.genevaux@univ-lemans.fr
}

a realistic non-linear model using a huge numer of parameters. Consequently, most of the works are based on an equivalent viscous coefficient's of a single degree of freedom model: it is adjusted to the experiment in one particular case [5].

The aim of this paper is to investigate experimentally the influence of several parameters on the modal damping coefficient above an acceleration's thresholds to induce relative movement between the beads [6]. The finality would be to create a adjustable equivalent viscous damping coefficient which could depend on the principle parameters of the system.

After having presented the experimental setup, this document will present the result of several parametric studies. First, the comparison between a not deformable mass and a grains is performed. Then, the sensitivity to a variation of the mass of grain and of the acceleration amplitude is studied. Finally, an observation of the modal damping's coefficient shows the efficiency of using grains to damp vibrations on the tested single degree of freedom system.

\section{Experimental setup}

In this study the behaviour of a single degree of freedom system is investigated (see Fig.1). It is composed of a rigid cavity whose mass is $m_{\text {cavity }}=3.140 \mathrm{~kg}$, guided in translation in the gravity direction and suspended on a stiffness. The granular material used is glass beads with a diameter of $\varnothing=2 \mathrm{~mm}$. This cavity set to vibration by the use of a shaker which excites the system with a sinusoidal 

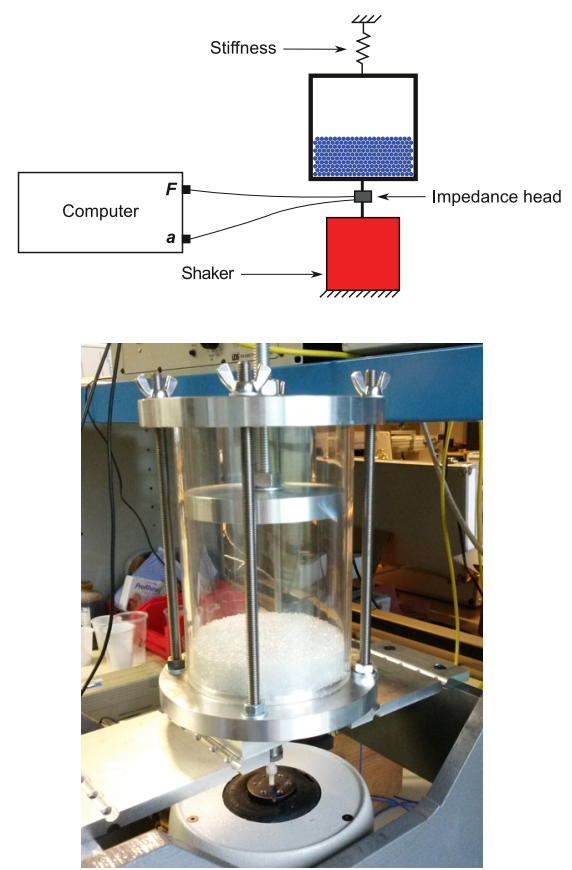

Figure 1. UP : Drawing of the setup - DOWN : Experimental set-up with granular media inside the cavity.

signal at an increasing step by step frequency. During each frequency increase, the acceleration amplitude is kept constant. An impedance head (B\&K 8001) is used to get the acceleration and force signal at the same point. The Frequency Response Function (FRF), in $\mathrm{kg}^{-1}$, is the ratio between acceleration and force.

Generally, the damping delivered by a plastic contact between two grains is considered as a friction damping. However the huge amount of grains can lead to an other interpretation (see Fig. 2).
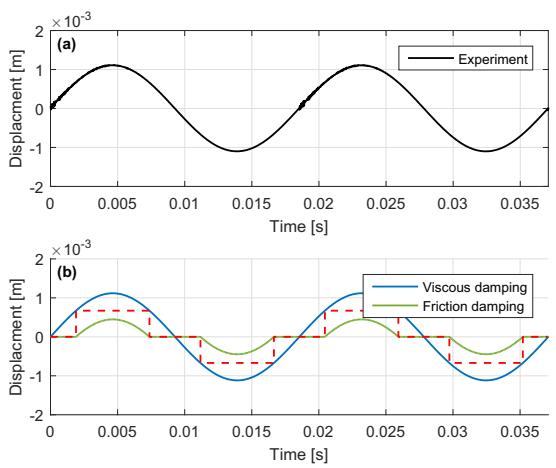

Figure 2. Influence of the several damping properties, comparison between viscous and frictional damping.

The figure 2-a shows a time capture of the container filled with grains. The figure 2-b shows a comparison in the time domain of the threshold effect where the friction force breaks and let the movement appears. The times without movement, can be called stick-times. This observation shows that no stick-times are present and allows us to consider an equivalent viscous damping induced by the amount of granular media. Indeed, if at the microscopic scale stick-times are present, they do not appear simultaneously in the whole volume. Therefore they are not detected by the sensors. The equivalent modal loss factor is also deduced by the circle fit method (see Fig.3). This method represents the complex FRF in a Nyquist graph.
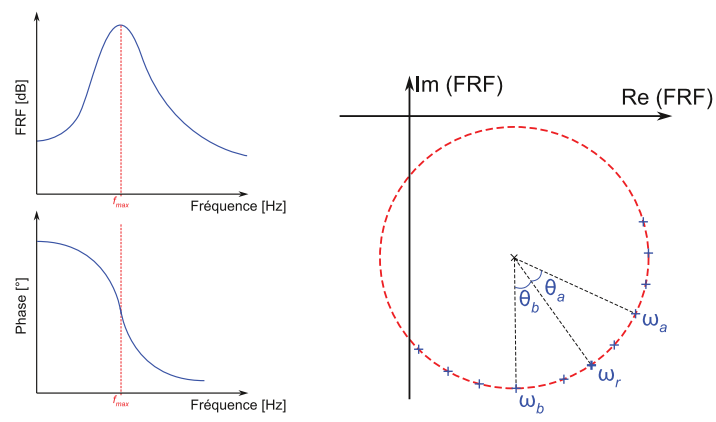

Figure 3. Frequency Response Function in the complex form.

The modal loss factor is deduced by the following equation [7] p.313 :

$$
\zeta=\frac{\omega_{a}^{2}-\omega_{b}^{2}}{2 \omega_{r}\left[\omega_{a} \tan \left(\theta_{a} / 2\right)+\omega_{b} \tan \left(\theta_{b} / 2\right)\right]} .
$$

\section{Experimental analysis}

\subsection{The use of granular media and its damping representation}

The figure 4 shows for a mass ratio of added material $r_{2}=\frac{m_{\text {material }}}{m_{\text {cavity }}}=15 \%$, only a small decrease of $1 \mathrm{~dB}$ of the maximal amplitude of the FRF if a rigid mass is used: this solution needs a huge ratio of added mass to achieve large decrease of amplitude.

By opposite, the use of a granular material with the same mass ratio is examined. As a matter of fact, even if the granular material is compacted, a $2 \mathrm{~dB}$ decrease of FRF amplitude is observed compared to the undeformable mass. Interaction between the grains is also acting in compacted volume of grain. Note that the quality factor appears to be constant between these two cases. This effect is increased when the granular material is loosed inside the cavity. In that case, a reduction of more than $20 \mathrm{~dB}$ of the amplitude of the FRF is achieved and the quality factor strongly decrease, indicating that damping occurs.

\subsection{Variation of the added mass on the primary system}

In a second part, the variation of added mass on the system is investigate. It has been shown previously that the not deformable mass was not the most efficient way to 


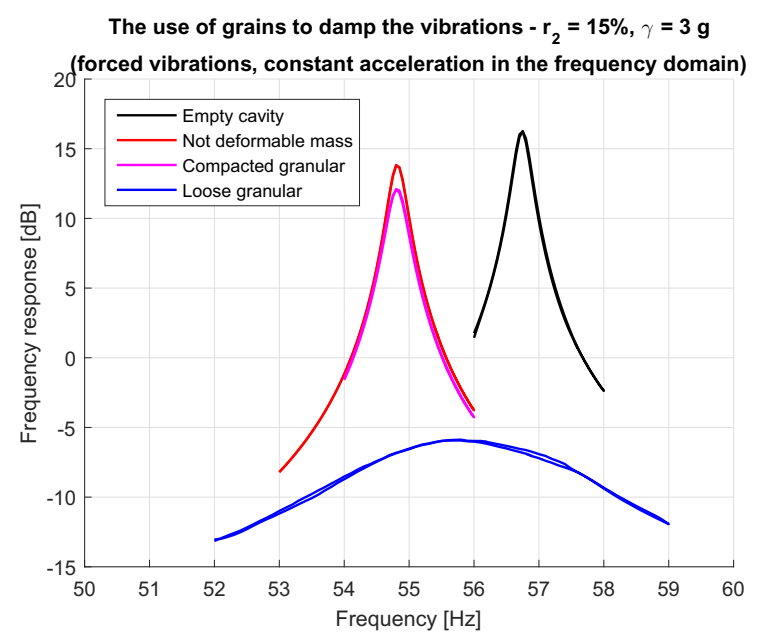

Figure 4. Comparison between the use of an inderformable mass and granular material $\left(\operatorname{ref}_{\mathrm{FRF}}=1 \mathrm{~kg}^{-1}\right)$.

damp the vibrations. In this part, two mass ratio are added separately with values of $r_{1}=5 \%$ and $r_{2}=15 \%$ and each are tested in two configurations: with compacted grains and with loose grains (see Fig.5).

Firstly, the behaviour of the compacted granular material is comparable with the behaviour of the added not deformable mass: when the mass increase, the resonance frequency $\omega_{0}$ declines, as shown in Eq. 2, where $\omega_{0}$ is the resonance frequency, $k$ the stiffness of the SDOF system and $m$ the mass of the SDOF.

$$
\omega_{0}=\sqrt{\frac{k}{m}} .
$$

However, with the same amount of granular material the non-compaction of the grain induces a huge damping. For example, with the mass ratio $r_{2}$ where the amplitude of the peak of the FRF is reduced of almost $15 \mathrm{~dB}$ for an acceleration $\gamma=3 g$, with $g$ the gravity acceleration. The loose granular material add grain interactions to the mass effect.

\subsection{Variation of the acceleration}

The use of a granular material leads to deal with nonlinearities according to the driving amplitude of acceleration $\gamma$. The higher amplitude of acceleration is, the higher dissipation becomes. A stiffening of the system can also be seen (see Fig. 6). Indeed, neither the contact forces between the grains are proportional to $\gamma$, nor the the mean stream of the grains in the bulk. With the same experimental setup by only increasing the amplitude of acceleration $\gamma$, the resonance peak decrease by nearly $15 \mathrm{~dB}$.

\subsection{Evolution of the modal damping coefficient}

The amount of damping is calculated by expression (Eq.1). This calculated value has to be compared to the empty system, which has no damping's treatment. The

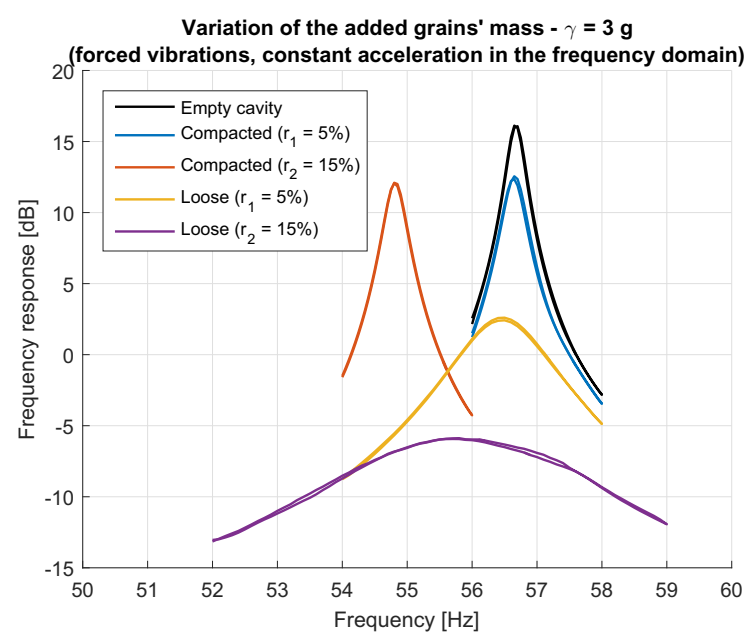

Figure 5. Variation of the grains' mass ratio on the resonanced system $\left(\mathrm{ref}_{\mathrm{FRF}}=1 \mathrm{~kg}^{-1}\right)$.

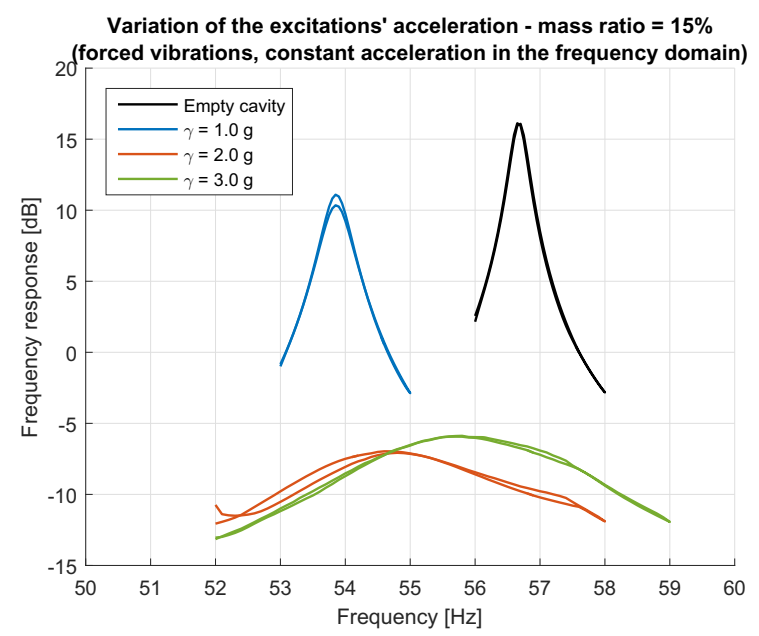

Figure 6. Variation of the acceleration's amplitude for a set-up with loose granular material $\left(\operatorname{ref}_{\mathrm{FRF}}=1 \mathrm{~kg}^{-1}\right)$.

results are presented with a grain's mass ratio $r_{1}=5 \%$ and $r_{2}=15 \%$ (see Fig.7).

The modal damping coefficient of the bare structure is rather equal from $0.3 \%$ to $0.4 \%$ independently of the acceleration. In the case of a mass ratio $r_{1}$, the different configurations don't show an important variation of the damping coefficient except with the Loose configuration where an variation is shown.

Moreover, the use of the mass ratio $r_{2}$ gives larger variation of the modal damping coefficient. Firstly, an increase of the measured damping appears above a threshold value of acceleration $\gamma_{t}=1 g$.

For loose granular media, the damping coefficient reach a high value $(4 \%)$, which reach up to 10 times the value obtained by the not deformable mass $(0.4 \%)$. By increasing the acceleration's amplitude above $2.5 \mathrm{~g}$, a 

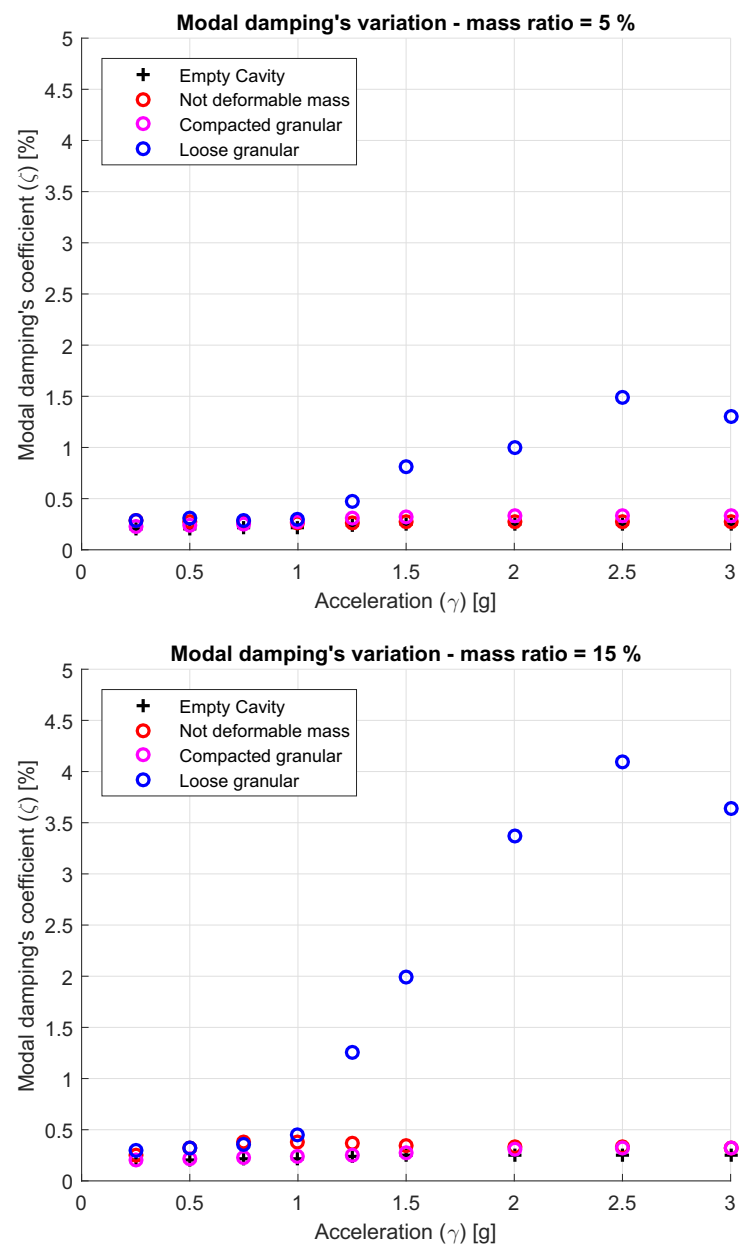

Figure 7. UP : System's modal damping, mass ratio $=5 \% /$ DOWN : System's modal damping, mass ratio $=15 \%$.

reduction of this coefficient is observed. At this particular value of acceleration, measured signals are no longueur sinus-like curves due to the hight number of particles interactions. This lead to a bias in the modal loss factor calculation. However the Energyloss (calculate with the help of the Lissajous method) continues to increase with the acceleration's amplitude.

Finally, the most efficient way to damp the vibrations is to have a cavity's volume much larger than the volume of the granular material to let the grains interact under the vibration and so dissipate the vibration's energy.

\section{Summary and conclusion}

This paper describes experimentally the effect of a granular media on the damping of a single degree of freedom system. Glass grains with a diameter of $\varnothing=2 \mathrm{~mm}$ are used. The studied system consist of a SDOF on which a cavity is fixed, inside which a granular media or a not deformable mass are placed.

To be efficient the granular media have to be not confined and the acceleration must be greater than $\gamma_{t}=1 \mathrm{~g}$. The more is the mass of granular media, the more efficient is the damping. For a mass ratio of $r_{2}=15 \%$ the added damping can be 9 time the damping of the bare system. Moreover, because the damping depends strongly on the acceleration's amplitude, the amount of granular material has to be optimised to obtain a chosen damping target.

\section{Acknowledgements}

This study is part of the VIBROLEG project managed by IRT Jules Verne (French Institute in Research and Technology in Advanced Manufacturing Technologies for Composite, Metallic and Hybrid Structures). The authors wish to associate the industrial and academic partners of this project; respectively Airbus, CETIM, Daher, DCNS Research, General Electrics, STX France, Bureau Veritas, le Laboratoire d'Acoustique de l'Université du Maine and l'Université du Maine.

\section{References}

[1] C.N. Bapat, S. Sankar, Journal of Sound and Vibration 99, 85 (1985)

[2] M. Saeki, Journal of Sound and Vibration 251, 153 (2002)

[3] M. Heckel, A. Sack, J.E. Kollmer, T. Pöschel, Physica A: Statistical Mechanics and its Applications 391, 4442 (2012)

[4] W. Liu, G.R. Tomlinson, J.A. Rongong, Journal of Sound and Vibration 280, 849 (2005)

[5] G. Michon, A. Almajid, G. Aridon, Journal of Sound and Vibration 332, 536 (2013)

[6] J.M. Génevaux, N. Dauchez, O. Doutres, Journal of Sound and Vibration 326, 150 (2009)

[7] D. Ewins, Modal testing: theory, practice and application - Second Edition, Mechanical engineering research studies: Engineering dynamics series (Research Studies Press Ltd., 2000), ISBN 9780863800177 